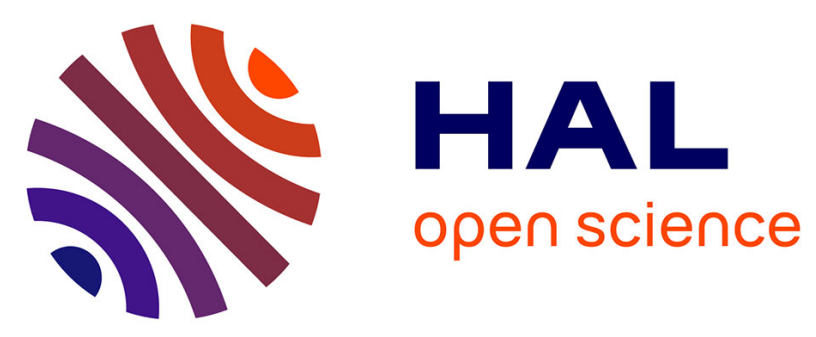

\title{
Self-consistent coupling of the three-dimensional fluid turbulence code TOKAM3X and the kinetic neutrals code EIRENE
}

Dongmei Fan, Yannick Marandet, Patrick Tamain, Hugo Bufferand, Guido Ciraolo, Philippe Ghendrih, Eric Serre

\section{To cite this version:}

Dongmei Fan, Yannick Marandet, Patrick Tamain, Hugo Bufferand, Guido Ciraolo, et al.. Self-consistent coupling of the three-dimensional fluid turbulence code TOKAM3X and the kinetic neutrals code EIRENE. Contributions to Plasma Physics, 2018, 58 (6-8), pp.490-496. 10.1002/ctpp.201700216 . hal-02114027

\section{HAL Id: hal-02114027 \\ https://hal-amu.archives-ouvertes.fr/hal-02114027}

Submitted on 29 Apr 2019

HAL is a multi-disciplinary open access archive for the deposit and dissemination of scientific research documents, whether they are published or not. The documents may come from teaching and research institutions in France or abroad, or from public or private research centers.
L'archive ouverte pluridisciplinaire HAL, est destinée au dépôt et à la diffusion de documents scientifiques de niveau recherche, publiés ou non, émanant des établissements d'enseignement et de recherche français ou étrangers, des laboratoires publics ou privés. 


\title{
Self-consistent coupling of the three-dimensional fluid turbulence code TOKAM3X and the kinetic neutrals code EIRENE
}

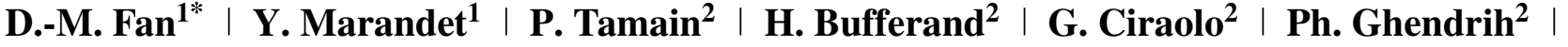 \\ E. Serre ${ }^{3}$
}

${ }^{1}$ PIIM, Aix-Marseille Univ., CNRS, Marseille, France

${ }^{2}$ IRFM, CEA, St. Paul-lez-Durance, France

${ }^{3}$ M2P2, Aix-Marseille Univ., CNRS, Marseille,

France

*Correspondence

D.-M. Fan, PIIM, Aix-Marseille Univ., CNRS,

Marseille F-13397, France.

Email: dong-mei.fan@univ-amu.fr

Funding Information

This research was supported by the A*MIDEX project KFC, ANR-11-IDEX-0001-02. Euratom

research and training programme $(\mathrm{H} 2020$

EURATOM), 633053. project Equip@Meso,

ANR-10-EQPX-29-01.

The three-dimensional (3D) turbulence code TOKAM3X-EIRENE, coupling the 3D non-isothermal version of TOKAM3X to the EIRENE Monte Carlo solver has been developed with the ability to simulate self-consistently the interactions between large-scale flows and turbulence both in limited and diverted plasmas, including recycling. This is especially important for diverted plasmas, where neutrals play a key role and where the recycling source is strongly dominant. The code package relies on the same interface as the Soledge2D-EIRENE code, which retains state-of-the-art plasma-wall interaction, as well as atomic and molecular physics. In this paper, we present the first results obtained in WEST divertor geometry, in laminar mode, with the aim of verifying the new code package. The divertor density regimes are recovered, and the code results are shown to be consistent with the results of the two-point model, thus opening the way for turbulent simulations.

\section{KEYWORDS}

density regimes, edge plasma transport, numerical modelling

\section{1 | INTRODUCTION}

Particle and power exhaust, that is, the removal of the helium ash and the fusion power without damage to the reactor itself, specifically its plasma-facing components (PFCs), is a key issue for next-step devices. The control of the deposited heat flux and erosion at the divertor target plates is a major issue for the design and operation of next-step, high-power, long-pulse devices such as the International Thermonuclear Experimental Reactor (ITER). ${ }^{[1]}$

Heat flux calculations presently rely on transport codes, which are a combination of two-dimensional (2D) fluid codes for charged particles and a kinetic Monte Carlo code modelling the neutral species behaviour as well as their interaction with the background plasma (e.g., SOLPS, ${ }^{[2]}$ SOLEDGE2D-EIRENE, ${ }^{[3,4]}$ EDGE2D ${ }^{[5]}$ ). All these codes solve mean-field equations in which the gradient diffusion hypothesis is applied to model turbulent fluxes. ${ }^{[6]}$ However, so far the anomalous transport coefficients introduced through this procedure are not consistently calculated, and are simply taken as the input parameters of the simulations. When used to interpret experiments, these transport coefficients are chosen so that upstream radial profiles match with the experimental profiles. As a result, these tools have limited predictive capabilities. Going beyond these mean-field models requires resolving turbulent fluctuations, in a global three-dimensional (3D) geometry, since strong interactions between mean/mesoscale flows and micro-turbulent fluctuations have been evidenced..$^{[7,8]}$ Going for global simulations (in the geometrical sense) also requires paying more attention to the forcing of the turbulence, especially for the charged particle source. In fact, the dominant particle source in the scrape-off layer (SOL)/edge is generally recycling, which occurs close to the divertor plates in diverted plasmas operated in the relevant regimes. Density profiles are thus expected to be mostly flat in the core (excluding pinches and/or neutral beam injection (NBI)-related sources in the plasma core). Compared to a situation where the particle 
source is located at the inner boundary condition of the simulation, this affects the pressure gradient in the edge region and thus the drive for interchange turbulence there. Recycling also strongly affects parallel flows in the SOL, especially in diverted configurations. ${ }^{[9]}$

Over the last decades, considerable simulation efforts have been dedicated to developing the 3D turbulence codes BOUT,$++{ }^{[10]} \mathrm{GBS}^{[11]}$ and TOKAM3X ${ }^{[12]}$ to investigate edge turbulent transport. This work relies on TOKAM3X, which includes both open and closed field lines in both limiter and diverted geometries. So far, the isothermal version of TOKAM3X has given new insights into the properties of edge/SOL turbulence, with first simulations in the X-point diverted geometry. ${ }^{[7,8]}$ However, in order to bring the simulations closer to experimental situations in divertor operation, it is necessary to include recycling physics in the models. This is mandatory to address regimes relevant for next-step machines, high recycling regimes, and even detached regimes, ${ }^{[13,14]}$ all the more because experimental evidence points towards changes of transport with the density regimes. ${ }^{[15]}$ Several groups have implemented neutral models in their code, either in $2 \mathrm{D}^{[16-18]}$ or $3 \mathrm{D}$ codes: a simplified kinetic model in $\mathrm{GBS}^{[19]}$ and a fluid model in BOUT++. ${ }^{[20]}$ Moreover, it has been pointed out that additional non-linearities introduced by neutral particle physics lead to additional closure issues in transport codes, ${ }^{[21-23]}$ which are difficult to address quantitatively in mean-field models. Building on the experience gained during the development of Soledge2D-EIRENE, the TOKAM3X code has been fully coupled to the EIRENE Monte Carlo solver. ${ }^{[24]}$ The resulting TOKAM3X-EIRENE code package is first described in this paper, the focus being on code verification.

The rest of the paper is organized as follows: in Section 2, we present the governing fluid equations and the physical setting including description of the geometry. In Section 3, we perform an upstream density scan to recover the density regimes in WEST-like diverted cases. Furthermore, we verify the computations by quantitatively comparing the modified two-point model (2PM) with those from TOKAM3X-EIRENE. The conclusion is provided in Section 4.

\section{2 | THE TOKAM3X-EIRENE CODE}

TOKAM3X is a 3D global transport and turbulence fluid code for the edge and SOL plasma of tokamaks, with full geometrical flexibility for the poloidal plasma equilibrium. The particle conservation equation, or continuity equation, is

$$
\partial_{t} N+\vec{\nabla} \cdot\left[N\left(u_{\|}+\vec{u}_{E}+\vec{u}_{\nabla B}^{e}\right)\right]=\vec{\nabla} \cdot\left(D_{N} \overrightarrow{\nabla_{\perp}} N\right)+S_{N} .
$$

The parallel momentum conservation equation is

$$
\begin{gathered}
\partial_{t} \Gamma_{i}+\vec{\nabla} \cdot\left[\Gamma_{i}\left(\frac{\Gamma_{i}}{N} \vec{b}+\vec{u}_{E}+\vec{u}_{\nabla B}^{i}+\vec{u}_{\text {curv }}^{i}\right)+N T_{i} \vec{b}\right] \\
=N T_{i} \vec{\nabla} \cdot \vec{b}-N \nabla_{\| \phi}-R_{\|}+\vec{\nabla} \cdot\left[D_{\Gamma} N \vec{\nabla}\left(\frac{\Gamma_{i}}{N}\right)\right]+\vec{\nabla} \cdot\left(D_{N} \frac{\Gamma_{i}}{N} \vec{\nabla} N\right)+S_{\Gamma},
\end{gathered}
$$

where $R_{\|}=0.71 N \nabla_{\|} T_{e}+\eta_{\|} N J_{\|}$and $E_{\|}=-\nabla_{\|} \phi$. The energy balance for electrons is

$$
\begin{gathered}
\partial_{t}\left(\frac{3}{2} p_{e}\right)+\vec{\nabla} \cdot\left[\frac{5}{2} T_{e}\left(\Gamma_{i}-J_{\| \mid}\right) \vec{b}+\frac{3}{2} p_{e}\left(\vec{u}_{E}+\vec{u}_{\nabla B}^{e}\right)\right]+\vec{\nabla} \cdot \vec{q}_{e} \\
=\left(J_{\|}-\Gamma_{i}\right) E_{\|}+u_{\| i} R_{\|}+Q_{\| e}+S_{E e}+\vec{\nabla} \cdot\left(D_{T e} N \vec{\nabla}_{\perp} T_{e}\right)+\vec{\nabla} \cdot\left(D_{N} \frac{3}{2} T_{e} \vec{\nabla}_{\perp} N_{i}\right),
\end{gathered}
$$

while for ions, it is

$$
\begin{gathered}
\partial_{t}\left(\frac{3}{2} p_{i}+\frac{\Gamma_{i}^{2}}{2 N}\right)+\vec{\nabla} \cdot\left[\left(\frac{5}{2} p_{i} \frac{\Gamma_{i}^{2}}{2 N}\right) u_{\| i} \vec{b}+\left(\frac{3}{2} p_{i}+\frac{\Gamma_{i}^{2}}{2 N}\right)\left(\vec{u}_{E}+\vec{u}_{\nabla B}^{i}+\vec{u}_{c u r v}\right)\right]+\vec{\nabla} \cdot \vec{q}_{i} \\
=N u_{\| i} E_{\|}-u_{\| i} R_{\|}+Q_{\| i}+S_{E i}+\vec{\nabla} \cdot\left(D_{T i} N \vec{\nabla}_{\perp} T_{i}\right)+\vec{\nabla} \cdot\left[D_{N}\left(\frac{3}{2} T_{i}+\frac{\Gamma_{i}^{2}}{2 N}\right) \vec{\nabla}_{\perp} N\right]+\vec{\nabla} \cdot\left[\Gamma_{i} D_{\Gamma} \vec{\nabla}\left(\frac{\Gamma_{i}}{N}\right)\right] .
\end{gathered}
$$

The charge conservation equation is

$$
\partial_{t} W+\vec{\nabla} \cdot\left(W \frac{\Gamma_{i}}{N} \vec{b}+W \vec{u}_{E}+W \vec{u}_{\nabla B}^{i}\right)=\vec{\nabla} \cdot N\left(\vec{u}_{\nabla B}^{i}-\vec{u}_{\nabla B}^{e}\right)+\vec{\nabla} \cdot\left(J_{\|} \vec{b}\right)+\vec{\nabla} \cdot\left(D_{W} \vec{\nabla} W\right)+S_{W},
$$

where $\vec{q}_{e}=-\chi_{\| e} \nabla_{||} T_{e} \vec{b}, \vec{q}_{i}=-\chi_{\| i} \nabla_{||} T_{i} \vec{b}$, and $W=\vec{\nabla} \cdot\left[\frac{N}{B^{2}} \vec{\nabla} \phi+\frac{1}{B^{2}} \vec{\nabla}\left(N T_{i}\right)\right] \cdot \vec{b}$ is a unit vector along the magnetic field, and $\eta$ is the normalized parallel collisional resistivity of the plasma. The energy exchange term between electrons and ions is denoted by $Q_{\| i}=-Q_{\| e}=3 \frac{m_{e}}{m_{i}} \frac{N}{\tau_{e}}\left(T_{e}-T_{i}\right)$. Equations (1)-(5) are, respectively, the particle balance equation, the parallel momentum balance equation, the energy balance equations, and the charge balance equation. The parallel Ohm's law with the electron terms neglected is given by $N_{e} E_{\|}=-\nabla_{\|} p_{e}+R_{\|}$. The derivation and normalization were carried out as in Ref. 12. In this work, the 


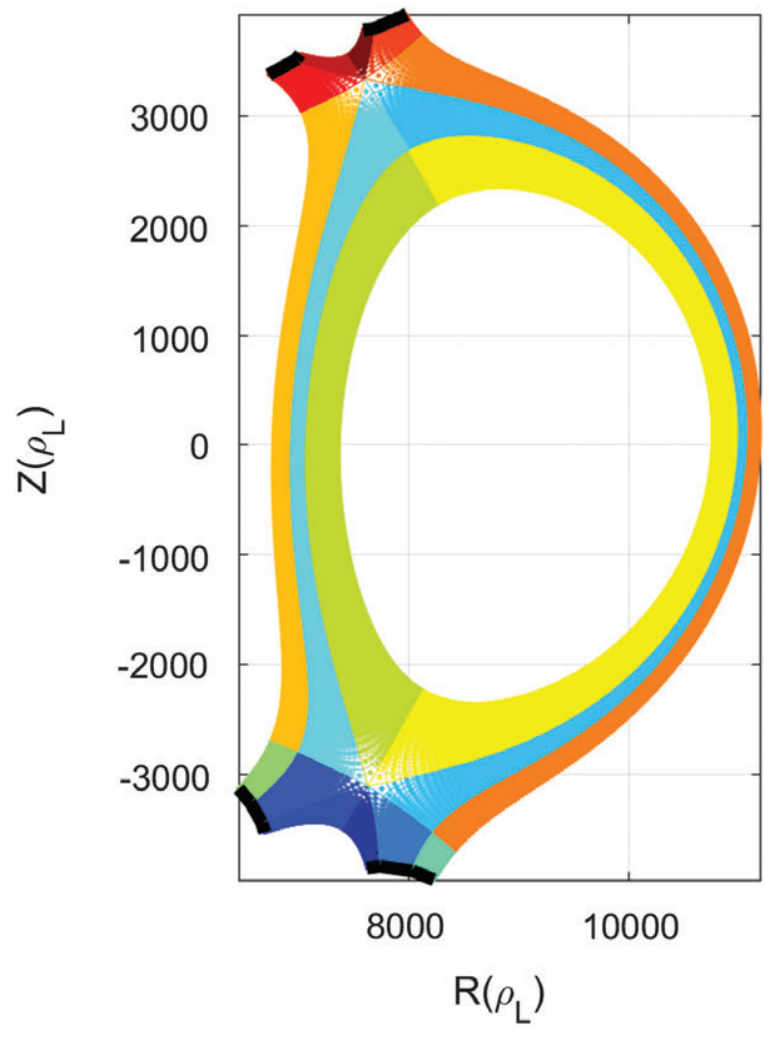

FIGURE 1 The WEST-like mesh grid used in TOKAM3X-EIRENE. Distances are expressed in Larmor radii $\rho_{L}$, with $\rho_{L}=0.256 \mathrm{~mm}$ here. The colours show the domain decomposition in use, with 16 zones

code is run in the non-isothermal mode, evolving the electron and ion temperatures, which play an important role in recycling physics. The magnetic field is fixed and given as the input of the code, and in the following a full-scale WEST equilibrium is used (Figure 1), together with a simplified wall/divertor target geometry retaining the WEST open divertor feature. The particle source term $S_{n}$, parallel momentum source term $S_{\Gamma}$, and energy source term $S_{E i / e}$ due to interactions with neutrals (atoms and/or molecules) are computed with the EIRENE code.

\section{1 | Coupling with the neutral code EIRENE}

In order to include neutral physics, the TOKAM3X code has been coupled to EIRENE, ${ }^{[24]}$ which is based on a Monte Carlo process. EIRENE tracks the test particles trajectories (atoms and molecules) and their interactions with the plasma electrons and ions, such as ionization, charge exchange, molecule dissociation, and backscattering of neutrals on the plasma surface. The coupling of the two codes entails data exchange (to be described below), but also physics issues, in particular at the sheath. All the developments available in Soledge2D-EIRENE, ${ }^{[4]}$ in particular to treat the sheath, are available to TOKAM3X-EIRENE since the two codes use the same interface, named STYX. The latter also manages the set-up of EIRENE in coupled runs. In terms of data exchanges, EIRENE takes the TOKAM3X ion flux profiles along the wall (more accurately, at the magnetized sheath entrance) and sample ions hitting the wall accordingly. The latter are then recycled into neutrals (atoms or molecules) according to TRIM databases for backscattering coefficients. The atoms or molecules thus created are followed in the plasma background calculated by TOKAM3X. The resulting particle, momentum, and energy sources are calculated accordingly, and handed back to TOKAM3X. Since TOKAM3X relies on a mixed implicit/explicit scheme, time steps are typically of the order

of $w_{c i}^{-1}\left(\omega_{c i}\right.$ is the ion gyro-frequency, i.e., $\left.\sim 10^{-8} \mathrm{~s}\right)$, and the EIRENE solver is not called at every time step of the fluid solver ("short cycling"). The STYX interface allows running EIRENE in the time-dependent mode, but this feature is not used here. This means that neutrals do not see any "time horizon", that is, they are tracked during times larger than the time step $\Delta t$ if they are not ionized or pumped after a time $\Delta t$. Note that actually using this feature in turbulent simulations will require careful tuning of the size of the buffer where neutrals reaching the time horizon are stored. Finally, we also mention that while TOKAM3X is a hybrid openmp/MPI code, EIRENE is parallelized with MPI only, a situation requiring further consideration.

\section{2 | Geometry and parameters}

The TOKAM3X code is designed to run with complex equilibria including $\mathrm{X}$-points. The magnetic field can be expressed as $\vec{B}=F_{0} R_{0} \vec{\nabla} \phi+\vec{\nabla} \Psi \times \vec{\nabla} \phi$, where $F_{0}$ is a total flux number, $\Psi$ is a poloidal flux function, and $\phi$ denotes the toroidal angle. 
(a)

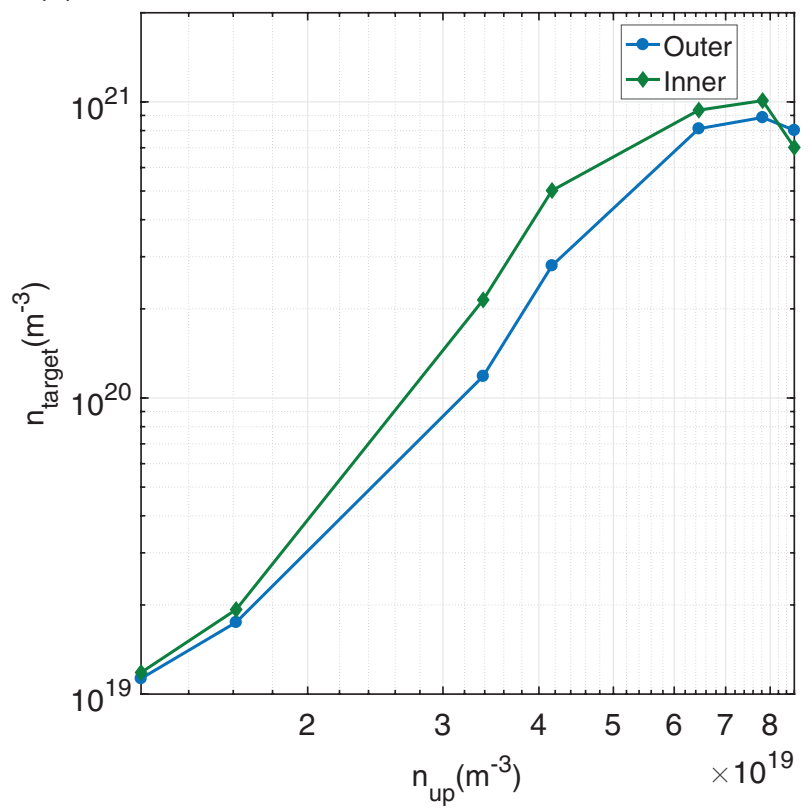

(b)

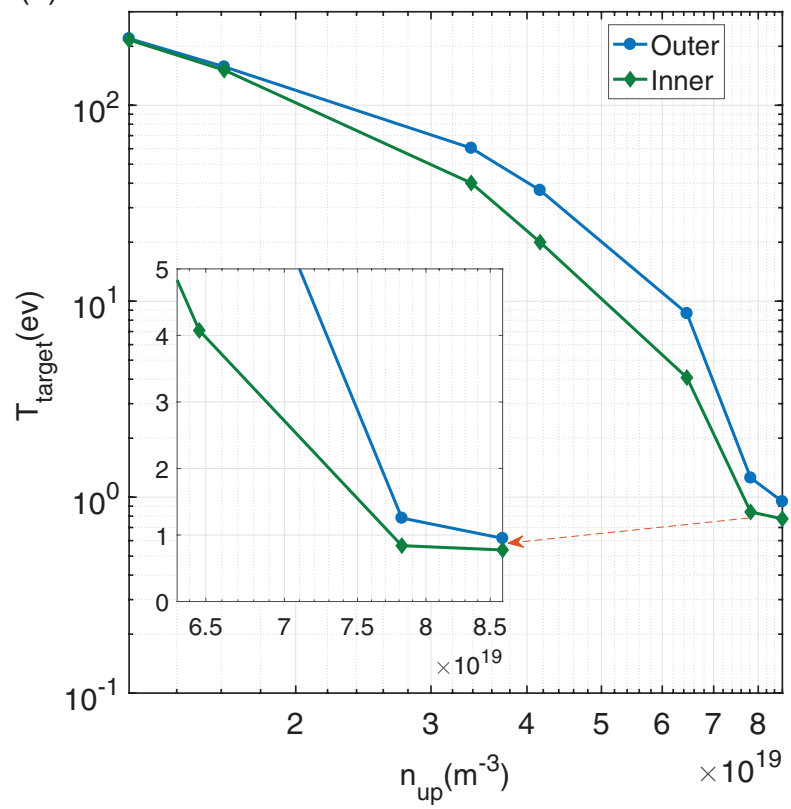

FIGURE 2 (a) Target plasma density $n_{t}$ and (b) temperature $T_{t}$ as a function of the outer upstream separatrix plasma density $n_{u p}$, for the inner and outer lower divertor

FIGURE 3 Dependence of total recycling flux in the inner and outer lower divertor legs as a function of the outer upstream separatrix density $n_{u p}$

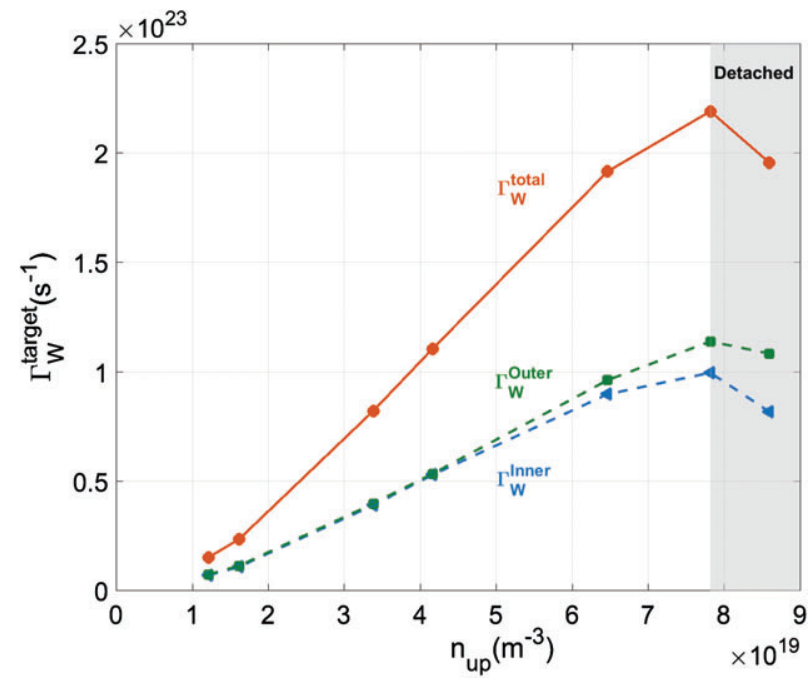

The function $\Psi$ is chosen so as to have a dimensionless magnetic field $B=1$ at the tokamak axis. The poloidal flux function has been taken from those prepared for the operation of WEST, with two X-points. The minor radius $a$ is equal to $1800 \rho_{L}$, where $\rho_{L}$ is the ion Larmor radius calculated for the reference parameters used to normalize the plasma fields $\left(n_{0}=2 \times 10^{19} \mathrm{~m}^{-3}, T_{0}=50 \mathrm{eV}\right.$, and $B_{0}=4 \mathrm{~T}$ ). The aspect radio $R / a=5.1$; that is, the major radius is $R=2.3 \mathrm{~m}$. The simulation domain extends from $r_{\min }=0.8 a$ in the closed field-lines region to $r_{\max }=1.2 a$. The diffusion coefficients for density, parallel momentum, and vorticity are set as $D_{N}=D_{\Gamma}=D_{W}=0.5 \mathrm{~m}^{2} \mathrm{~s}^{-1}\left(4 \times 10^{-2}\right.$ in dimensionless units, the normalization factor being $\rho_{L}^{2} \omega_{c i}$, again calculated for the reference parameters). They are uniform in the simulation volume. The parallel resistivity is given as $\eta_{\|}=1 \times 10^{-5} \frac{B_{0}}{e n_{0}}$. The total input power in the present simulations is $1.4 \mathrm{MW}$, which is equally shared between ions and electrons, and the power influx at the core edge interface is modelled by a Gaussian source of width $25 \rho_{L}$. In the simulations presented here, there is a particle source at the core, but ultimately the code will be run using a gas puff as primary particle source. The flux of atoms reaching the core edge interface is added to this source, with a uniform poloidal distribution. The outermost magnetic flux surface is assumed to have an effective recycling coefficient (albedo) of $R=0.99$. Since the focus is on divertor regimes, the code is run with a deuterium plasma without drifts, and the electrostatic potential is calculated in the adiabatic approximation. Furthermore, the code is run in 2D mode (axisymmetry is enforced by conservative averaging in the toroidal direction), hence producing laminar (i.e., non-turbulent) plasma solutions. The effective diffusion coefficients $\left(D_{N / \Gamma / W}\right)$ modelling turbulent transport in these laminar simulations are prescribed as input data. The code is thus run in exactly the same way as a transport code as a 


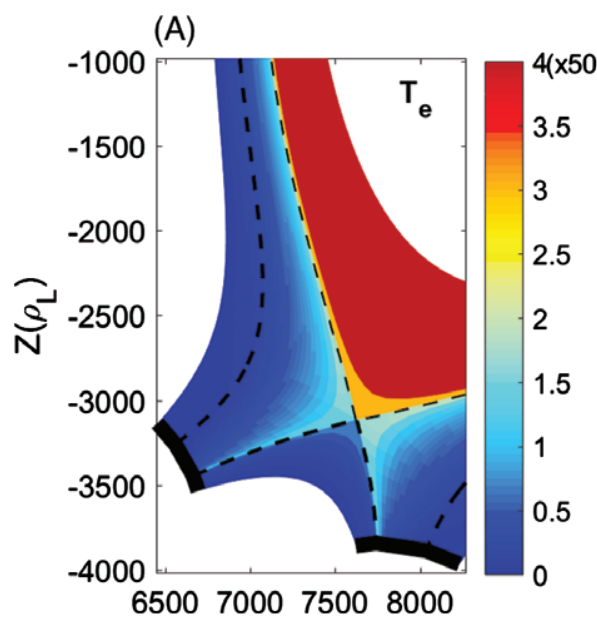

(B)

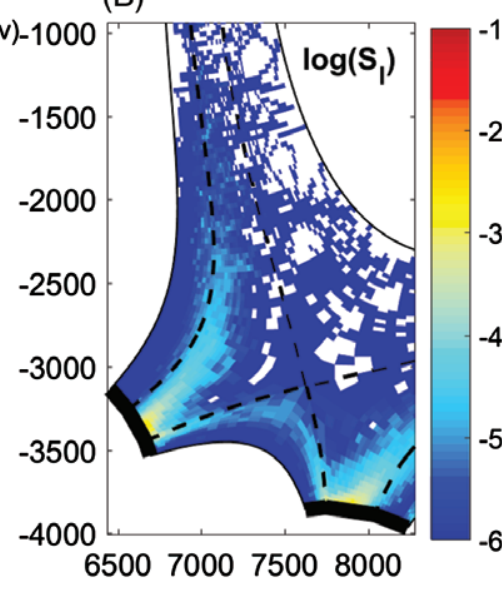

(C)

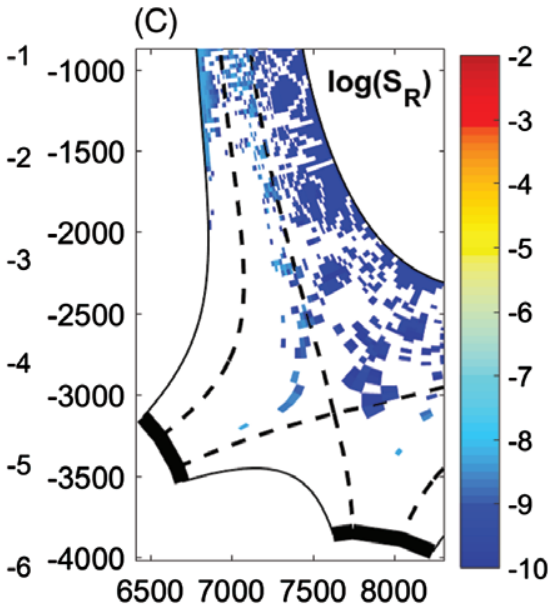

(D)

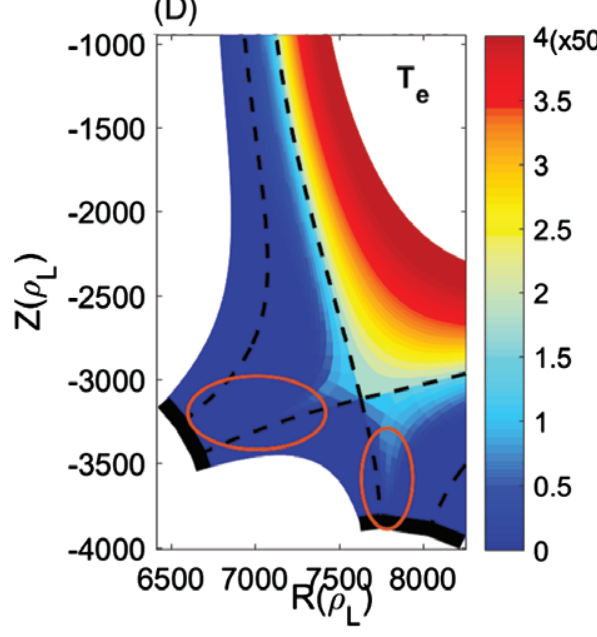

(E)

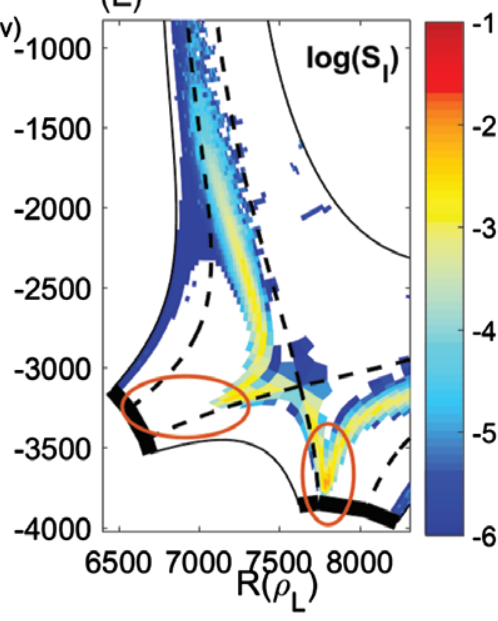

$(\mathrm{F})$

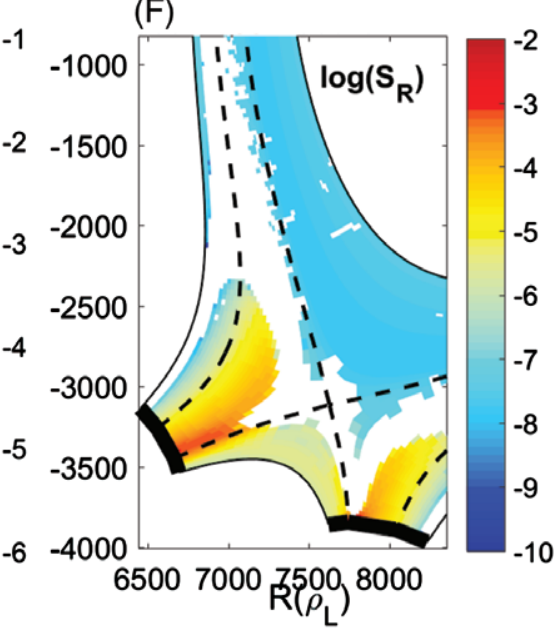

FIGURE 4 TOKAM3X-EIRENE simulation results. Electron temperature $\left(T_{e}\right)$, ionization source $\left(\log \left[S_{I}\right]\right)$, and recombination sinks $\left(\log \left[S_{R}\right]\right)$ in the attached regime (a)-(c) and detached regime (d)-(f)

first but key step of the verification procedure. Convergence towards a steady-state solution (in fact, a statistically stationary state due to statistical noise from EIRENE ${ }^{[25]}$ ) is assessed by checking for stationarity of all the fields and verifying the global particle and energy balances in the simulations. We thus check that the flux absorbed in the wall $\left(\phi_{\text {wall }}=R\left(\phi_{D^{+}}+\phi_{D}+\phi_{D_{2}} / 2\right)\right)$ plus the neutral flux at the core edge interface are equal to the influx from the core sustaining the simulation (i.e., the integral of the forcing). As far as energy is concerned, we calculate the energy flux transferred to the wall, which includes the net (i.e., incident minus reflected) kinetic energy flux from both plasma and neutrals, the recombination energy flux on the surface, and the radiated flux, and compare it with the total input power. For simulations presented here, the agreement is within a few percent, both for particles and energy.

\section{3 | RESULTS OF CALCULATION AND MODEL VERIFICATION}

The purpose of this section is to show, first qualitatively, that the code correctly reproduces density regimes as the upstream density increases. The scan is made by increasing the particle source in the core from $5.2 \times 10^{19}$ to $1.3 \times 10^{22} \mathrm{part} / \mathrm{s}$, keeping the power constant at 1.4 MW. Figure 2 shows the evolution of the target density $n_{t}$, measured in the outer divertor at the separatrix as a function of upstream mid-plane density. The latter ranges from $10^{19}$ to $9 \times 10^{19} \mathrm{~m}^{-3}$. Note that, based on Greenwald density considerations, it is not expected that the separatrix density can rise above $\sim 3.5 \times 10^{19} \mathrm{~m}^{-3}$ in WEST. However, as previously noted, this work is devoted to verifying the code package, so we ignore such concerns. As the upstream density increases, so does the target density $n_{t}$ in both lower divertor legs, while the target temperature $T_{t}$, very close to the upstream temperature $T_{u}$ at lowest $n_{u}$, decreases strongly. For the highest upstream density, the temperature is down to $\sim 1 \mathrm{eV}$, hence corresponding to the onset of detachment. This behaviour is thus qualitatively in line with common wisdom on divertor regimes, showing the transition from the sheath limited to the high recycling and even detached regime. A divertor imbalance is observed, with the inner divertor showing higher density/lower temperature in all the attached cases. The latter results from 
FIGURE 5 Temperature ratio $T_{t} / T_{u}$ as a function of the upstream separatrix plasma density $n_{u p}$ obtained from the simulation (blue line with circles) compared to the standard (orange stars) and modified two-point model (2PM) scalings (green diamonds and red triangles are, respectively, the results from the modified 2PM [with $f_{r}$ ] and from the modified 2PM [with $f_{r}$ ] taking into account the loss factors)

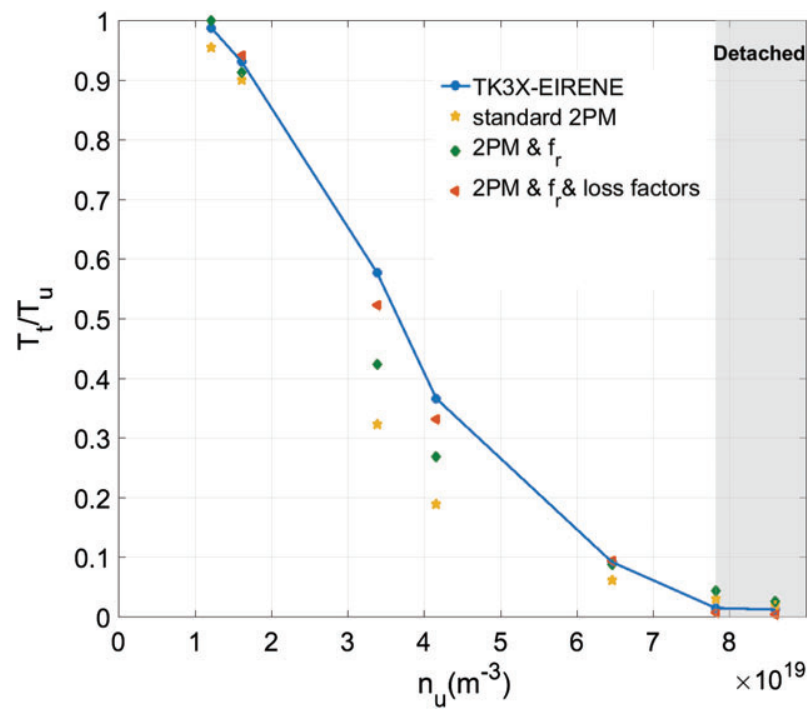

the low-field-side/high-field-side asymmetry of the magnetic configuration (e.g., Ref. 26). These results are confirmed by the evolution of the total recycling flux in both legs as a function of upstream density, shown in Figure 3, showing a roll-over at a separatrix density of $7.7 \times 10^{19} \mathrm{~m}^{-3}$. With increasing $n_{u p}$, as shown in Figure 4, recombination becomes stronger (Figure 4f) and the cold plasma region in the divertor extends towards the X-point (Figure 4d). Virtually, the divertor is occupied by the cold dense plasma, and the plasma ionization source and the recombination sink are localized close to the X-point and the targets, respectively.

In order to verify whether these trends are quantitatively correct, we compare the results with the $2 \mathrm{PM}^{[9]}$ of the SOL in the following. The $2 \mathrm{PM}$ connects the upstream and downstream densities and temperatures, using (a) (total) pressure conservation, that is, $n_{u} T_{u}=2 n_{t} T_{t}$, (b) parallel energy balance, and (c) sheath physics to express the parallel heat flux as function of the target temperature, namely $q_{t}=\gamma n_{t} c_{s} T_{t}$. Here, $\gamma$ is the total sheath heat transmission coefficient, and $c_{s}=\left(2 T_{t} / m_{i}\right)^{1 / 2}$ is the sound speed. The total sheath heat transmission coefficient is taken to be $\gamma=7$, in accordance with the values used in the code. Power

balance leads to $T_{u}^{7 / 2}=T_{t}^{7 / 2}+\frac{7}{2} \frac{q_{t} L}{\kappa_{0}}$, where $\kappa_{0}$ is the electron conductivity ( $\kappa_{0}=2000$ when electron temperature is expressed in $\mathrm{eV}$ ) and $L$ the parallel connection length between the upstream location and the target location. The latter is estimated by $L \simeq \pi q R$. Correction factors ${ }^{[9]}$ are usually introduced to account for volumetric momentum (resp. power) losses, $f_{\text {mom }}$ (resp. $\left.f_{\text {pow }}\right)$. $f_{\text {mom }}$ describes the effects resulting from interactions with neutrals, that is, for momentum losses, friction and volume recombination. The latter can be measured from the simulations. We here assume that all the power is conducted $\left(f_{\text {cond }}=1\right)$. Further corrections can be made to account for the total flux expansion, ${ }^{[27]}$ leading to the following expression valid when $T_{t} \ll T_{u}$ (in fact, $T_{t}^{7 / 2} \ll T_{u}^{7 / 2}$ ):

$$
\frac{T_{t}}{T_{u}}=\left(\frac{7}{2} \frac{q_{\|} L}{\kappa}\right)^{8 / 7}\left(\frac{4 \kappa}{7 L \gamma n_{u}}\right)^{2}\left(\frac{\ln f_{r}}{f_{r}-1}\right)^{-6 / 7} \frac{f_{\text {mom }}^{2} f_{\text {cond }}^{6 / 7}}{\left(1-f_{\text {pow }}\right)^{2}},
$$

where $f_{r}$ is the total magnetic flux expansion. $q_{\|}$is no longer constant along the flux tube $\left(q_{\|} \propto B\right)$, thus $q_{\|}=q_{t} f_{r}$. Here, $f_{r} \simeq 0.7$ so that accounting for flux expansion leads to a correction factor of $\sim 1.4$ for $T_{t} / T_{u}$ (green diamonds as shown in Figure 5). Note that with reasonable values for our simulations, namely $f_{\text {mom }}=0.8, f_{\text {cond }}=1$, and $f_{\text {pow }}=0.2$, we have $f_{\text {mom }}^{2} f_{\text {cond }}^{6 / 7} /\left(1-f_{\text {pow }}\right)^{2}=1$. When $T_{t}$ is not small compared to $T_{u}$, we solve for the full model. Comparisons to the $2 \mathrm{PM}$ allow checking whether the transition from the sheath-limited regime to the high-recycling regime occurs in the correct range of upstream density $n_{u}$, for the power input chosen. The values obtained for $T_{t} / T_{u}$ in the simulations (using the outer mid-plane data) are compared with those from the $2 \mathrm{PM}$ in Figure 5, and they show good agreement. The $2 \mathrm{PM}$ calculations assume here $f_{\text {mom }}=0.8, f_{\text {cond }}=1$, and $f_{\text {pow }}=0.1$ in the attached regime, and $f_{\text {mom }}=0.2, f_{\text {cond }}=1$, and $f_{\text {pow }}=0.5$ in the detached regime (red triangles shown in Figure 5), in accordance with pressure losses observed in the simulations. The agreement shows that the code predictions for the transition to the conduction-limited and high-recycling regimes are in the correct $n_{u}$ range.

\section{4 | CONCLUSIONS}

The coupling of the TOKAM3X 3D turbulence code with the Monte Carlo code EIRENE has been verified in the laminar mode in X-point geometry, clearing the way for simulations of global electrostatic plasma turbulence including state-of-the-art neutral particle physics. The TOKAM3X-EIRENE verification has been carried out by analysing the results of a density scan, where 
standard divertor regimes are observed. The results of the code are consistent with 2PM considerations, for which we considered different refinements. Importantly, the "attached regime" and the "detached regime" are recovered, the total recycling flux along the wall showing a roll-over at very high density case, when the electron temperature in the divertor reaches $\sim 1 \mathrm{eV}$.

\section{ACKNOWLEDGMENTS}

D.M.F. has been funded through the A*MIDEX project (ANR-11-IDEX-0001-02), the latter being funded by the Investissements d'Avenir French Government programme. This work has been carried out within the framework of the EUROfusion Consortium and French Research Federation for Fusion Studies and has received funding from the Euratom research and training programme 2014-2018 under grant agreement No. 633053. The views and opinions expressed herein do not necessarily reflect those of the European Commission. This work was granted access to the HPC resources of Aix-Marseille Université financed by the project Equip@Meso (ANR-10-EQPX-29-01) of the programme "Investissements d'Avenir" supervised by the Agence Nationale pour la Recherche.

\section{REFERENCES}

[1] A. Loarte, B. Lipschultz, A. S. Kukushkin, G. F. Matthews, P. C. Stangeby, N. Asakura, G. F. Counsell, G. Federici, A. Kallenbach, K. Krieger, A. Mahdavi, V. Philipps, D. Reiter, J. Roth, J. Strachan, D. Whyte, R. Doerner, T. Eich, W. Fundamenski, A. Herrmann, M. Fenstermacher, P. Ghendrih, M. Groth, A. Kirschner, S. Konoshima, B. LaBombard, P. Lang, A. W. Leonard, P. Monier-Garbet, R. Neu, H. Pacher, B. Pegourie, R. A. Pitts, S. Takamura, J. Terry, E. Tsitrone, the ITPA Scrape-off Layer and Divertor Physics Topical Group, Nucl. Fusion 2007, 47, S203.

[2] R. Schneider, X. Bonnin, K. Borrass, D. Coster, H. Kastelewicz, D. Reiter, V. Rozhansky, B. Braams, Contrib. Plasma Phys. $2006,46(1-2), 3$.

[3] H. Bufferand, B. Bensiali, J. Bucalossi, G. Ciraolo, P. Genesio, P. Ghendrih, Y. Marandet, A. Paredes, F. Schwander, E. Serre, J. Nucl. Mater. 2013, 438, S445.

[4] H. Bufferand, G. Ciraolo, Y. Marandet, J. Bucalossi, P. Ghendrih, J. Gunn, N. Mellet, P. Tamain, R. Leybros, N. Fedorczak, F. Schwander, E. Serre, Nucl. Fusion 2015, 55, 053025 .

[5] R. Simonini, G. Corrigan, G. Radford, J. Spence, A. Taroni, Contrib. Plasma Phys. 1994, 34(2-3), 368.

[6] H. Bufferand, G. Ciraolo, P. Ghendrih, Y. Marandet, J. Bucalossi, C. Colin, N. Fedorczak, D. Galassi, J. Gunn, R. Leybros, E. Serre, P. Tamain, Contrib. Plasma Phys. 2016, 56(6-8), 555.

[7] D. Galassi, P. Tamain, H. Bufferand, G. Ciraolo, P. Ghendrih, C. Baudoin, C. Colin, N. Fedorczak, N. Nace, E. Serre, Nucl. Fusion 2017, $57,036029$.

[8] P. Tamain, P. Ghendrih, H. Bufferand, G. Ciraolo, C. Colin, N. Fedorczak, N. Nace, F. Schwander, E. Serre, Plasma Phys. Controlled Fusion 2015, 57, 054014.

[9] P.C. Stangeby, The Plasma Boundary of Magnetic Fusion Devices, Institute of Physics Publishing, London 2000.

[10] B. Dudson, M. Umansky, X. Xu, P. Snyder, H. Wilson, Comput. Phys. Commun. 2009, 180, 1467.

[11] P. Ricci, F. Halpern, S. Jolliet, J. Loizu, A. Mosetto, A. Fasoli, I. Furno, C. Theiler, Plasma Phys. Controlled Fusion 2012, $54,124047$.

[12] P. Tamain, H. Bufferand, G. Ciraolo, C. Colin, D. Galassi, P. Ghendrih, F. Schwander, E. Serre, J. Comput. Phys. 2016, 321 , 606.

[13] S. I. Krasheninnikov, A. Kukushkin, W. Lee, A. Pshenov, R. Smirnov, A. Smolyakov, A. Stepanenko, Y. Zhang, Nucl. Fusion 2017, 57(10), 102010.

[14] B. Lipschultz, B. LaBombard, J. Terry, C. Boswell, I. Hutchinson, Fusion Sci. Technol. 2007, 51(3), 369.

[15] M. Agostini, J. L. Terry, P. Scarin, S. J. Zweben, Nucl. Fusion 2011, 51, 053020.

[16] Y. Marandet, P. Tamain, R. Futtersack, P. Ghendrih, H. Bufferand, P. Genesio, A. Mekkaoui, J. Nucl. Mater. 2013, 438, S518.

[17] N. Bisai, R. Jha, P. K. Kaw, Phys. Plasmas 2015, 22, 022517.

[18] A. Thrysoe, L. Tophoj, V. Naulin, J. J. Rasmussen, J. Madsen, A. Nielsen, Plasma Phys. Controlled Fusion 2016, 58, 044010.

[19] C. Wersal, P. Ricci, Nucl. Fusion 2015, 55, 123014.

[20] J. Leddy, B. Dudson, H. Willett, Nucl. Mater. Energy 2017, 12, 994.

[21] E. Havlickova, W. Fundamenski, V. Naulin, J. J. Nielsen, S. Wiesen, J. Horacek, J. Seidl, J. Nucl. Mater. 2011,415, S471.

[22] Y. Marandet, A. Mekkaoui, D. Reiter, P. Boerner, P. Genesio, J. Rosato, H. Capes, F. Catoire, M. Koubiti, L. Godbert-Mouret, R. Stamm, Plasma Phys. Controlled Fusion 2011, 53, 065001.

[23] F. Guzman, Y. Marandet, P. Tamain, H. Bufferand, G. Ciraolo, P. Ghendrih, R. Guirlet, J. Rosato, M. Valentinuzzi, Plasma Phys. Controlled Fusion 2015, 57, 125014.

[24] D. Reiter, M. Baelmans, P. Boerner, Fusion Sci. Technol. 2005, 47(2), 172.

[25] Y. Marandet, H. Bufferand, G. Ciraolo, P. Genesio, P. Meliga, J. Rosato, E. Serre, P. Tamain, Contrib. Plasma Phys. 2016, 56, 604.

[26] P. Ghendrih, T. Auphan, B. Bensiali, M. Bilanceri, K. Bodi, A. Bonnement, H. Bufferand, G. Chiavassa, G. Ciraolo, R. Futtersack, C. Guillemaut, Y. Marandet, A. Mentrelli, D. Moulton, A. Paredes, R. Pasquettie, E. Serre, P. Tamain, J. Nucl. Mater. 2013, 438, S368.

[27] T. Petrie, J. Canik, C. Lasnier, T. Petrie, A. Leonard, M. Mahdavi, J. Watkins, M. Fenstermacher, J. Ferron, R. Groebner, D. Hill, Nucl. Fusion 2013, 53 , 113024. 